Conclusions LSTs were predominant in the proximal colon, but a greater proportion of LST-GM, greater mean-size, and greater presence of submucosal cancer found in rectal LST. LST-GM and LST-NGPD had a higher incidence of intramucosal carcinoma and submucosal carcinoma, in particular, LSTNGPD was highest rates of submucosal invasion, should be noticed by endoscopic physicians.

\section{IDDF2018-ABS-0160 ENDOSCOPIC CHARACTERISTICS, TREATMENT AND FOLLOW-UP OF COLORECTAL LATERALLY SPREADING TUMOURS}

Zilin Kan*, Peng Jin. PLA Army General Hospital, China

\subsection{6/gutjnl-2018-IDDFabstracts.134}

Background To analyse the endoscopic characteristics and therapeutic methods of colorectal laterally spreading tumours.

Methods Colorectal laterally spreading tumours were collected from digestive endoscopy centre of PLA army general hospital from Mar 2010 to May 2016. Their relationship between morphology, predilection site, pit patterns and histopathology were analysed retrospectively. And compared the follow-up results of different endoscopic treatment.

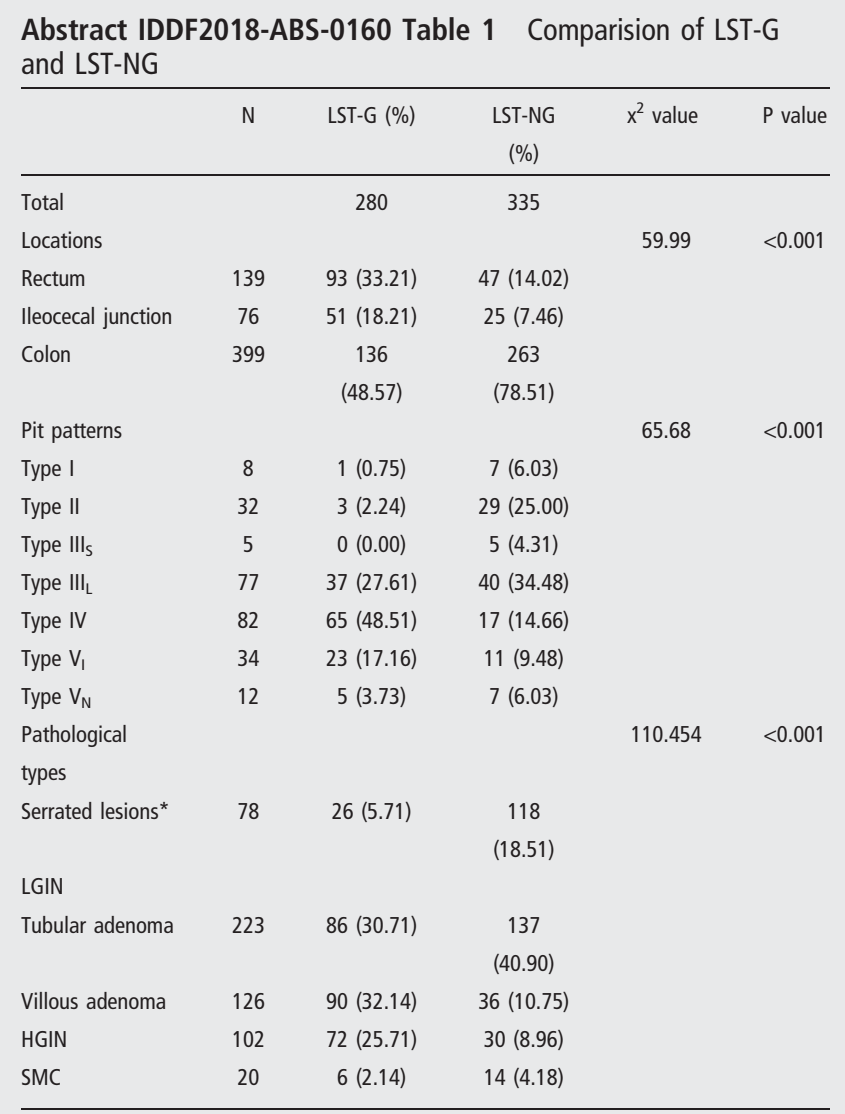

*Serrated lesions: a group of lesions characterised by serrated epithelium, including hyperplastic polyp(HP), traditional serrated adenoma (TSA) and sessile serrated adenoma/polyps (SSA/Ps). LGIN, low grade intraepithelial neoplasia; HGIN, high grade intraepithelial neoplasia; SMC, submucosal carcinoma.

Results A total of 615 colorectal laterally spreading tumours (551patients) were collected. Granular (LST-G) was detected more frequently in the ileocecal region and rectum (75/140, 53.7\%);
Non-granular (LST-NG) were preferentially located in the rest part of the colon. Pit patterns type IV has large proportion in the LST-G and type IIIL in the type LST-NG. On histopathology, LST - NG in the majority with tubular adenoma (40.9\%), while the LST - G than LST - NG more villiform than LST - NG $(32.14 \%$ vs $10.75 \%)$. LST-G and LST-NG had a higher incidence of intramucosal carcinoma and submucosal carcinoma, 27.86\% (78/280) and $13.13 \%$ (44/250), respectively. LST-NG was highest rates of submucosal invasion: $4.18 \%$. To follow-up after treatment 330 cases of patients, 285 cases of lost to follow-up. Follow-up time span of 1 76 months, the median for 15 months. A total of 10 cases of recurrence, endoscopic piecemeal mucosal resection (EPMR) higher recurrence rate was 13.79\% (4/29), compared to nearly 3 years after the ESD treatment, there is no recurrence of LST.

Conclusions Had a higher incidence of intramucosal carcinoma and submucosal carcinoma, in particular, LST-NGPD was highest rates of submucosal invasion, should be noticed by endoscopic physicians. The endoscopic submucosal dissection (ESD) is safe and effective treatment.

\section{IDDF2018-ABS-0164 THE TEMPORAL RELATIONSHIP OF DAILY LIFE STRESS, EMOTIONS AND BOWEL SYMPTOMS IN IRRITABLE BOWEL SYNDROME: A SMARTPHONE-BASED EXPERIENCE SAMPLING STUDY}

${ }^{1}$ Yawen Chan*, ${ }^{1}$ Suzanne Ho-wai So, ${ }^{2}$ Arthur Dun Ping Mak, ${ }^{2}$ Kewin Tien Ho Siah, ${ }^{1}$ Wai Chan, ${ }^{3}$ Justin Che-yuen Wu. 'Department of Psychology, The Chinese University of Hong Kong, Hong Kong; ' Department of Psychiatry, The Chinese University of Hong Kong, Hong Kong; ${ }^{3}$ Department of Medicine and Therapeutics, The Chinese University of Hong Kong, Hong Kong

\subsection{6/gutjnl-2018-IDDFabstracts. 135}

Background The current study aimed to model the moment-tomoment relationship between daily life stress, negative emotions and bowel symptoms among patients with irritable bowel syndrome - diarrhoea subtype (IBS-D) in the flow of daily life using a smartphone-based experience-sampling method (ESM).

Methods Patients with IBS-D $(n=27)$ and healthy controls $(\mathrm{HC})(\mathrm{n}=30)$ completed ESM ratings of their real-time daily life stress, which was defined as the subjective stress related to daily activities, negative emotion and bowel symptoms 8 times a day for 14 consecutive days, following a baseline interview measuring bowel and mood symptoms. Moment-to-moment association between ESM variables was tested within and between groups using multilevel regression modelling.

Results Patients with IBS-D reported more severe bowel symptoms ( $p s<0.01$ ) than HCs, but levels of daily life stress and negative affect were comparable between groups ( $p s>0.05$ ). Time-lagged analysis of ESM data revealed that, among patients with IBS-D, daily life stress predicted a decrease in abdominal pain and urgency to defecation at a subsequent time point ( $p s<0.05$ ), whereas severity of bowel symptoms and occurrence of diarrhoea predicted a subsequent increase in negative affect and daily life stress $(p s<0.05$ ). The above associations were not found among HCs (figure 1).

Conclusions ESM unveiled the dynamic relationship between bowel symptoms, stress and emotionality. Patients with IBS-D responded to bowel symptoms with more stress and distress momentarily. Counter-intuitively, daily life activity stress served as a protective factor for bowel symptoms. Our data informed the psychological understanding of IBS. 